\title{
Small scale structure in molecular gas from multi-epoch observations of HD $34078^{\star}$
}

\author{
E. Rollinde ${ }^{1}$, P. Boissé ${ }^{1,2}$, S. R. Federman ${ }^{3}$, and K. Pan ${ }^{3}$ \\ 1 Institut d'Astrophysique de Paris, 98 bis boulevard d'Arago, 75014 Paris, France \\ 2 Radioastronomie, UA CNRS 336, École Normale Supérieure, 24 rue Lhomond, 75231 Paris Cedex 05, France \\ 3 Department of Physics and Astronomy, University of Toledo, Toledo, OH 43606, USA
}

Received 22 November 2002 / Accepted 15 January 2003

\begin{abstract}
We present spectroscopic observations of the runaway reddened star HD 34078 acquired during the last three years at Observatoire de Haute Provence and McDonald Observatory as well as other spectra obtained since 1990. The drift of the line of sight through the foreground cloud due to the large transverse velocity of HD 34078 allows us to probe the spatial distribution of $\mathrm{CH}, \mathrm{CH}^{+}, \mathrm{CN}$ and DIBs carriers at scales ranging from about $1 \mathrm{AU}$ up to $150 \mathrm{AU}$. In particular, time variations in the equivalent width of absorption lines are examined. A few past and recent high resolution observations of $\mathrm{CH}_{\text {and }} \mathrm{CH}^{+}$ absorption are used to search for line profile variations and to convert equivalent widths into column densities.

The data set reveals a $20 \%$ increase in $\mathrm{CH}$ column density over the past 10 years with no corresponding variation in the column density of $\mathrm{CH}^{+}$or in the strengths of the 5780 and $5797 \AA$ DIBs. CN observations indicate that its excitation temperature has significantly increased from $<3.1 \mathrm{~K}$ in 1993 to $3.6 \pm 0.17 \mathrm{~K}$ in 1998 while the $\mathrm{CN}$ column shows only a modest rise of $\approx 12 \pm 6 \%$. The data also strongly suggest the existence of weak correlated variations in $\mathrm{CH}$ and $\mathrm{CH}^{+}$columns over periods of 6-12 months (or $\approx 10 \mathrm{AU}$ ).

These results are discussed in relation to $\mathrm{CH}^{+}$production mechanisms. A dense newly intervening clump is considered in order to explain the long-term increase in the column density of $\mathrm{CH}$, but such a scenario does not account for all observational constraints. Instead, the observations are best described by $\mathrm{CH}^{+}$production in a photodissociation region, like that suggested for the Pleiades and IC 348.
\end{abstract}

Key words. ISM: molecules - stars: individual: HD 34078 - ISM: structure

\section{Introduction}

While the existence of AU-scale structure is relatively well established in atomic gas (see e.g., Dieter et al. 1976; Diamond et al. 1989; Frail et al. 1994; Lauroesch et al. 1999; Faison \& Goss 2001; Welty \& Fitzpatrick 2001), the reality of such tiny fluctuations within molecular gas is still questionable. Indeed, only minor species can be easily observed towards molecular clouds and their spatial distribution might not reflect that of $\mathrm{H}_{2}$ if "chemical structure" were present. $\mathrm{H}_{2} \mathrm{CO}, \mathrm{HCO}^{+}$and $\mathrm{OH}$ apparently display column density fluctuations reaching 5 to $15 \%$ along lines of sight separated by about $10 \mathrm{AU}$ (Marscher et al. 1993; Moore \& Marscher 1995; Liszt \& Lucas 2000), while dust grains appear to be more smoothly distributed (Thoraval et al. 1996). At larger scales (about $10000 \mathrm{AU}$ ), Pan et al. (2001) find significant differences in $\mathrm{CN}, \mathrm{CH}$ and $\mathrm{CH}^{+}$absorption lines.

If marked enough, small scale structure within molecular gas might notably affect its time evolution, fragmentation and

\footnotetext{
Send offprint requests to: E. Rollinde, e-mail: rollinde@iap. fr

$\star$ Based on observations made at Observatoire de Haute Provence (France) and McDonald Observatory.
}

then utimately, star formation. It is therefore important to characterise the properties of such media, identify the parameters displaying fluctuations and quantify their amplitude and scalelength. To this aim, we have selected a bright 09.5 runaway star, HD 34078 (AE Aur), seen through a translucent cloud with $E(B-V)=0.52$ (Diplas \& Savage 1994). Thanks to its large transverse velocity, about $100 \mathrm{~km} \mathrm{~s}^{-1}$ for an assumed distance of $530 \mathrm{pc}$, the comparison of spectra taken at one year intervals provides a measurement of column density variations at a scale of $17 \mathrm{AU}$ (assuming a cloud distance of $400 \mathrm{pc}$ : Brown et al. 1995). To complement ongoing FUSE observations designed to investigate in a direct way small scale variations of the $\mathrm{H}_{2}$ column density (and then study density structure within molecular gas), we have undertaken repeated ground-based observations of $\mathrm{CH}, \mathrm{CH}^{+}$and $\mathrm{CN}$ absorption lines and diffuse interstellar bands (DIBs) towards HD 34078.

These optical spectra complement the FUSE UV data in several important respects:

- since $\mathrm{CH}$ is seen to correlate well with $\mathrm{H}_{2}$ over large scales (e.g., Federman 1982), it should be a good indicator of cloud structure. On the other hand, the abundance of $\mathrm{CH}^{+}$is largely independent of $\mathrm{H}_{2}$ column density, $N\left(\mathrm{H}_{2}\right)$; the large abundance 
of this species is still poorly understood and hot pockets of gas (e.g., shocks or vortices as proposed by Falgarone \& Puget 1995) could be the formation site. Then, $\mathrm{CH}^{+}$observations may reveal the structure of these presumably very small regions where energy is actively dissipated. Finally, CN depends nonlinearly on $\mathrm{H}_{2}$ and is thus an indicator of gas density (Federman et al. 1994).

- Given the brightness of HD 34078, high $S / N$ spectra can be obtained easily to search for very small fluctuations which would be difficult to detect in FUSE spectra.

- Spectral data in the visible range can be acquired much more easily than in the far UV, and therefore, it is possible to control the time (i.e., spatial) sampling and to accumulate a larger number of spectra. Moreover, several visible observations of HD 34078 have been performed in the past 10 years, thus giving access to large scale variations. A broad range of scales can then be explored through visible observations.

- High resolution observations providing information on the velocity distribution and its possible variations are feasible in the visible, but not in the FUSE range.

In this paper, we present observations made specifically for this project together with a comparison with older data. The whole set of spectra is used to search for time variable absorption and infer the structure implied for $\mathrm{CH}, \mathrm{CH}^{+}, \mathrm{CN}$ and diffuse bands carriers. Section 2 presents the observations and the methods used to analyse the data. In Sect. 3, we give the results obtained for $\mathrm{CH}, \mathrm{CH}^{+}, \mathrm{CN}$ and some selected DIBs. Since most observations have been obtained at low resolution, we mainly consider equivalent width variations. High resolution observations of $\mathrm{CH}$ and $\mathrm{CH}^{+}$are then used to infer velocity distributions and translate variations in equivalent width into column density variations for these two species. The implication of our results for the existence of AU-scale structure in the distribution of these tracers is discussed in Sect. 4.

The analysis of Cycle 1 and Cycle 2 FUSE data will be presented in a separate paper (Boissé et al. 2002; see also Boissé et al. 2001 and Le Petit et al. 2001 for a preliminary report). Our ultimate goal is to correlate the variations seen for $\mathrm{H}_{2}$ and those of other tracers like $\mathrm{CH}, \mathrm{CH}^{+}$and $\mathrm{CN}$ in order to improve the description of the structure.

\section{The data}

\subsection{The available spectra}

Spectra acquired at various observatories have been used for this study. In the following, these are named according to the observer and year of data taking. We obtained specifically for this project a series of nine spectra at the Observatoire de Haute Provence (OHP) (R99-02). In addition, high resolution spectra were recently recorded at McDonald Observatory (F02). The motivation was twofold. First, as we are interested in column density variations, we need to translate the observed changes in equivalent width, $W_{\lambda}$, into changes in $N$ (i.e., determine $N$ and $\left.\mathrm{d} N / \mathrm{d} W_{\lambda}\right)$. Since the lines are not optically thin, the conversion requires a good knowledge of the velocity distribution. This point is especially important for $\mathrm{CH} \lambda 4300$ because this feature is in fact a blend of two transitions and the analysis can

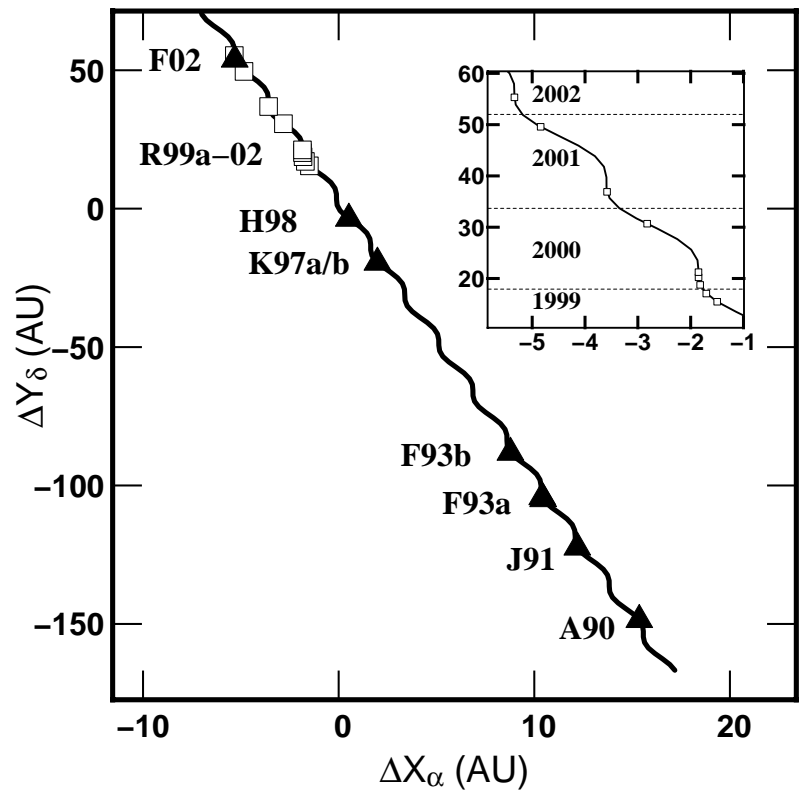

Fig. 1. The impact point of the line of sight towards HD 34078 onto the foreground cloud versus time. The motions of the earth, the sun and HD 34078 have been taken into account. Markers specify the position of the star for each epoch at which spectra were obtained (cf. Table A.1). Note that the $X$ and $Y$ scales are not identical. The insert focuses on the recent observations made for this project (R99a-R02, F02) that probe the smallest scales.

be notably affected by the splitting if the $b$ parameter is less than $2 \mathrm{~km} \mathrm{~s}^{-1}$ (Lien 1984). Second, only high resolution observations allow us to determine whether or not line shapes have changed, and they possibly reveal profile variations not associated with equivalent width changes.

HD 34078 was observed several times since 1991 by one of us (S. Federman at McDonald Observatory: F93a, F93b). Spectra of HD 34078 were also acquired by M. Allen (KPNO: A91), P. Jenniskens and F.X. Désert (at OHP: J91), J. Krelowski, G. A. Galazutdinov \& F. A. Musaev (at Terskol Observatory: K97a, K97b) and G. Herbig (at the Keck telescope: H98) who kindly provided their data, so that we could analyze them in the same way as our own and search for variations by direct comparison of the spectra. Note that in his study, Herbig (1999) searched for changes in line position with respect to the spectrum obtained by Adams (1949) and thus investigated the velocity structure of the foreground gas; no significant variation was observed.

Table A.1 summarises all these observations and gives the date, resolution and signal-to-noise ratio. The transitions (from $\mathrm{CH}, \mathrm{CH}^{+}, \mathrm{CN}$ or DIBs) contained in the observed wavelength range are indicated. These data altogether provide a sort of $1 \mathrm{D}$ cut through the cloud, the sampling of which is illustrated by Fig. 1. Below, we give some details concerning the observations of HD 34078 used in this paper and not previously published. 


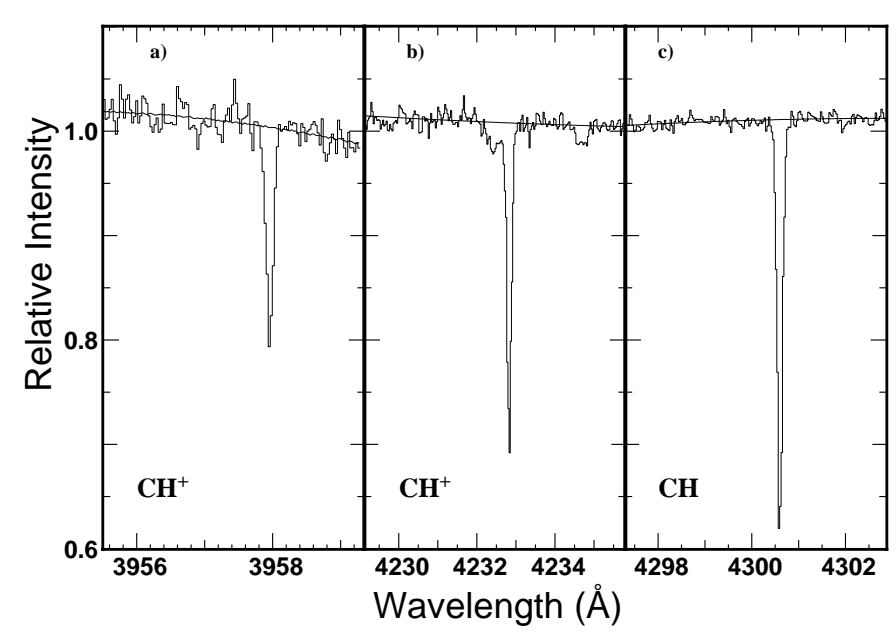

Fig. 2. The $\mathrm{CH}^{+}$lines at 3957 and $4232 \AA$ a,b) and $\mathrm{CH}$ line at $4300 \AA$ c) in the OHP spectrum recorded in December 1999. The raw spectrum has just been divided by a constant to scale the mean intensity to 1 . Note the broad Fe II stellar feature blended with the $4232 \AA$ Aine. The $S / N$ is 230 per $0.03 \AA$ pixel around $4300 \AA$.

\subsection{OHP observations}

The spectra were obtained using the fiber-fed echelle spectrograph Elodie (Baranne et al. 1996) mounted on the $1.93 \mathrm{~m}$ telescope (R99-02). The resolution is about 32000 . Initially, a spectrum was taken each month in order to probe very small scales. Since this series did not reveal significant variations, only two spectra were taken during the next winters. The range covered is $3906 \AA-6811 \AA$ and includes lines from $\mathrm{CH}(4300 \AA), \mathrm{CH}^{+}$(3957 and $4232 \AA$ ) and DIBs. For all spectra, the integration time is about 1 hour, split in 2 to 3 successive exposures of 30 or 20 minutes each in order to check the stability of the instrument and robustness of our analysis on independent spectra. The corresponding $S / N$ ratio is typically 150 per resolution element. Some examples of $\mathrm{CH}$ and $\mathrm{CH}^{+}$lines detected in OHP spectra are shown in Fig. 2. The spectra have been extracted and wavelength calibrated using the automatic on-line data reduction program attached to the instrument (see Baranne et al. 1996 for details); the wavelength calibration is based on thorium lamp spectra.

\subsection{McDonald observations}

Three data sets were acquired since the last published results (Federman et al. 1994) by one of us (S.R.F.). Two of the new sets utilized the 6 foot camera and the 2dcoude spectrograph on the $2.7 \mathrm{~m}$ telescope. A standard observing strategy, with bias frames, flat fields, and a Th-Ar comparison spectrum, was employed (see Knauth et al. 2001 for details of similar measurements). Individual orders containing $\mathrm{CH}^{+} \lambda 4232$ and $\mathrm{CH} \lambda 4300$ were imaged with the 6 foot camera in 1993. The nominal resolution was $1.5 \mathrm{~km} \mathrm{~s}^{-1}$. In 2002, spectra of CN, $\mathrm{CH}$ and $\mathrm{CH}^{+}$were acquired at a single setting of the 2 dcoude spectrograph. Here, the resolution was about $1.9 \mathrm{~km} \mathrm{~s}^{-1}$. Also in 1993, the Sandiford echelle spectrograph on the $2.1 \mathrm{~m}$ telescope (McCarthy et al. 1993) was used for measurements on various species in a similar fashion. One setting, centered on $3990 \AA$, provided data on $\mathrm{CN}, \mathrm{CH} \lambda \lambda 3878,3886,3890$ and $\mathrm{CH}^{+}$13957. The second setup at $4300 \AA$ measured absorption from $\mathrm{CH}^{+} \lambda 4232$ and $\mathrm{CH} \lambda 4300$. The widths of Th-Ar lines indicated a resolution of $\sim 7.5 \mathrm{~km} \mathrm{~s}^{-1}$ for these spectra. All data were reduced and analyzed with the IRAF package.

\subsection{Analysis of the data}

In most of the available HD 34078 spectra, the profiles are not resolved. We shall then look primarily for variations in $W_{\lambda}$.

We have been particularly careful in placing the continuum in a consistent manner for all epochs and also in estimating properly the uncertainty in $W_{\lambda}$. For some species, several lines are observed, e.g., at $3957 \AA$ and $4232 \AA$ for $\mathrm{CH}^{+}$. Similarly, a few observations of the $\mathrm{CH}$ lines at 3878,3886 and $3890 \AA$ are available for comparison with the $4300 \AA$ results. This redundancy is useful as a means to assess the robustness of our analysis and validate the error estimates.

We have developed an automatic procedure based on the MIDAS package alice in order to fit the continuum in a userindependent way and to measure $W_{\lambda}$ values. Several "clean" windows (generally two, located on both sides of the line) are selected; a polynomial fit with a degree ranging from 1 to 3 and depending on the shape of the spectrum is then performed. This provides a good estimate of the continuum in the interval covered by the absorption feature (see Fig. 2). One $\sigma$ uncertainties in $W_{\lambda}$ can be estimated as the quadratic sum of $\epsilon_{\mathrm{n}}$ and $\epsilon_{\mathrm{c}}$, the pixel-to-pixel noise and the uncertainty in the continuum level in the normalised spectrum respectively. The latter are given by

$\epsilon_{\mathrm{n}}=\sigma_{F} \times \delta \lambda \times \sqrt{N}$, and
$\epsilon_{\mathrm{c}}=\Delta \lambda_{\text {line }} \times|1-F|$,

where $\Delta \lambda_{\text {line }}$ is the total width of the absorption feature, $\delta \lambda$ is the pixel size and $N$ the number of pixels covered by the line $\left(\Delta \lambda_{\text {line }}=N \delta \lambda\right)$. The average (Eq. (2)) and the rms (Eq. (1)) of the normalised flux, $F$, are computed along the windows adjacent to the line. The robustness of the fitting procedure and the estimates for uncertainty have also been checked against synthetic spectra including absorption lines with known $W_{\lambda}$, a signal-to-noise ratio comparable to that in our data, and various continuum shapes.

Some transitions present difficulties. $\mathrm{CH}^{+} \lambda 4232$ is blended with a shallow stellar feature (from Fe II, see Fig. 2b) and the above procedure cannnot be applied. Similar problems are encountered with the $R(0) \mathrm{CN}$ line at $3876 \AA$. In such cases, the $W_{\lambda}$ is measured using the command integrate/line from MIDAS (an order 2-3 is chosen for the polynomial used to fit the spectrum immediately adjacent to the line) and the uncertainties are determined from successive measurements.

The high resolution profiles have been fitted using VPFIT (Carswell et al. 1987). No attempt was made to look for velocity shifts (the question addressed by Herbig 1999).

\section{Results}

We now present the results obtained for $\mathrm{CH}, \mathrm{CH}^{+}, \mathrm{CN}$ and some selected DIBs. For $\mathrm{CH}$ and $\mathrm{CH}^{+}$, we first discuss the homogeneous set of data obtained after November 1999 which 
probe time scales ranging from one month to 2.5 years (corresponding to 1.5-40 AU). The whole set of available observations made in the past 11 years are presented next. Finally, we discuss the high resolution data and their implications for velocity structure and column density variations.

\subsection{Structure in $\mathrm{CH}$}

\subsubsection{Small scale variations}

Figure 3 a displays the evolution of the equivalent width as a function of time for the $4300 \AA$ line. The variation since 1999 is fitted with a linear form $W_{\lambda}=W_{\lambda, 0}+\alpha\left(t-t_{\text {med }}\right)$ where $t_{\text {med }}$ is the median of observing times (December 2000). The data points are weighted according to signal-to-noise ratio in all fits performed in this paper. The contours of the reduced $\chi^{2}\left(W_{0}, \alpha\right)$ are plotted in Fig. 4a (dashed lines) for the linear case. A constant value is rejected only at the $1 \sigma$ level. The best fit, $W_{0}=56.6 \mathrm{~m} \AA$ and $\alpha=0.98 \mathrm{m \AA} \mathrm{yr}-1$ corresponds to $\Delta W_{\lambda} \simeq 2.2 \mathrm{~m} \AA$ between November 1999 and February 2002. It is overplotted in Fig. 3a (dotted line). Large variations at this scale are excluded since $-1.8<\Delta W_{\lambda}<6.3 \mathrm{m \AA}$ at the $3 \sigma$ level.

The linear fit is not fully satisfactory since residuals correspond to $2 \sigma$. This suggests that small additional erratic variations in $W_{\lambda}$ may be present. The observed amplitude is $1.50 \mathrm{~m} \AA$ (rms) about the linear fit (and $1.56 \mathrm{m \AA}$ about a constant value; the maximum difference is $4.8 \mathrm{m \AA}$ ). A Kolmogorov-Smirnov test (Miller 1956) shows that these values are too small compared to observational errors to indicate with certainty that we are detecting fluctuations in $W_{\lambda}(\mathrm{CH})$. However, the reality of the latter is supported by the fact that similar variations are observed for $\mathrm{CH}^{+}$lines (see below).

\subsubsection{Large scale variations}

Results for the full set of observations are given in Table A.2 and shown also in Fig. 3a. A significant increase is apparent. Fitting again with a linear variation (with $t_{\text {med }}=$ April 1996) gives a total increase of $\Delta W_{\lambda}=8 \mathrm{~m} \AA$ or $16 \%$ between 1992 and November 2001, consistent with the slope derived from the small scale data alone (see Fig. 4). The $3 \sigma$ limit is $4.2<\Delta W_{\lambda}<$ $13 \mathrm{~m} \AA$. However, the fit is even less satisfactory than for the recent data alone since the minimum $\chi^{2}$ is larger than $3 \sigma$. The assumption of a linear increase is somewhat arbitrary and visual inspection of Fig. 3 suggests that a step function might also provide an acceptable fit. The minimum $\chi^{2}$ is not significantly lowered, $\sim 2.6 \sigma$. The date at which the step occurs is well constrained by K97 and H98 measurements. The amplitude $\left(\Delta W_{\lambda} \sim 7 \mathrm{m \AA}\right)$ is similar to the total increase inferred from the linear fit. To assess the reality of an increase in $W_{\lambda}(4300)$ regardless of any assumption on its form, we performed a Pearson test to investigate the correlation between $W_{\lambda}$ and time. An uncorrelated evolution of $\mathrm{CH} \lambda 4300$ with time is rejected at the $4 \sigma$ level.

Other $\mathrm{CH}$ lines at 3886 and $3890 \AA$ have been observed in 1990, 1993 and 1998. Their variation should be consistent with the increase seen in $\mathrm{CH}$ 14300. Given the weakness of
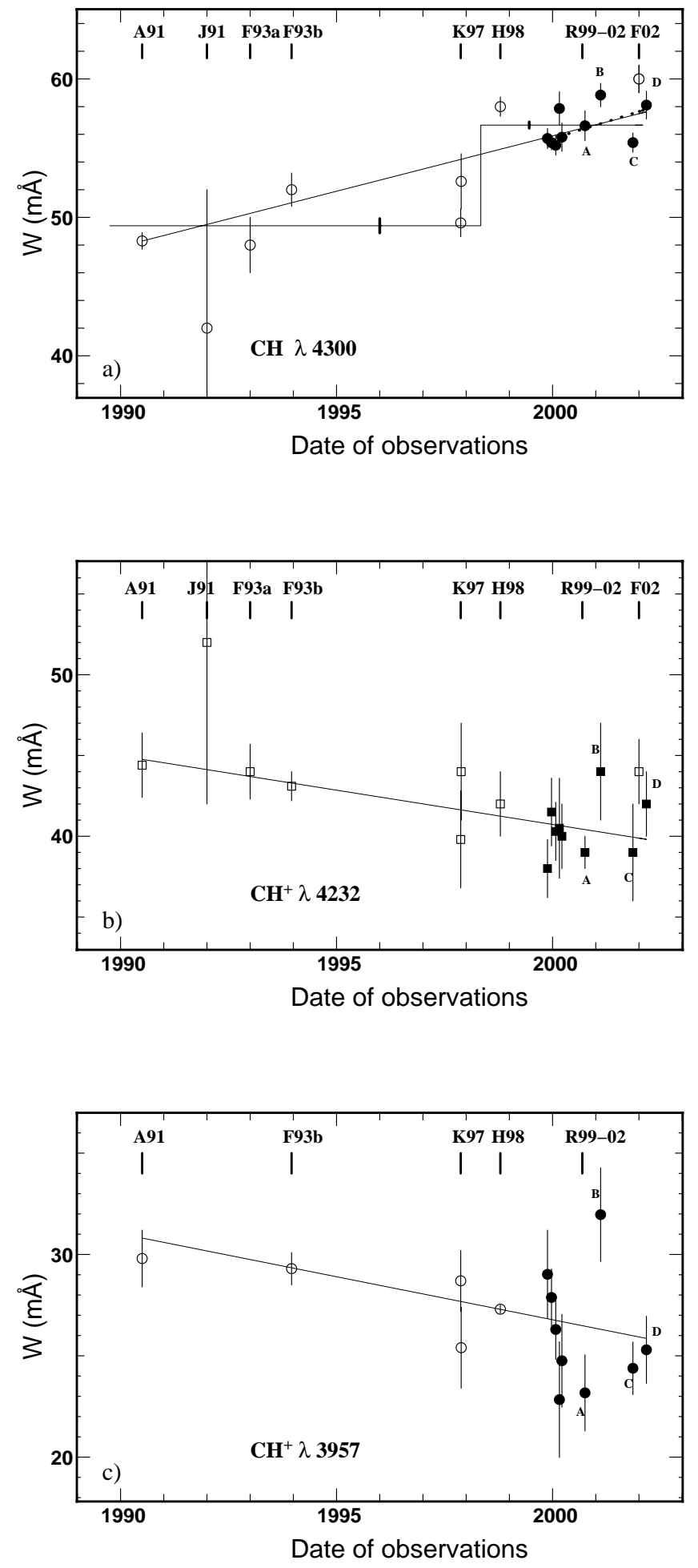

Fig. 3. The equivalent width of $\mathrm{CH}$ and $\mathrm{CH}^{+}$lines versus time. Top panel: $\mathrm{CH}$ 14300; middle panel: $\mathrm{CH}^{+}$14232, bottom panel: $\mathrm{CH}^{+}$13957. Solid markers show recent closely-spaced OHP observations. A linear fit is performed on the whole data set (solid lines). For recent OHP/F02 data on $\mathrm{CH} \lambda 4300$, a separate linear fit is given (dotted line on top panel). A fit by a step function is also shown for the $\mathrm{CH}$ data, with errors for the upper and lower values indicated by the small vertical bars. Note the similarity between $\mathrm{CH}$ and $\mathrm{CH}^{+}$variations from October 2000 to March 2002 (points labelled A, B, C and D), suggesting the reality of correlated erratic fluctuations. Such fluctuations are also suggested by the McDonald data of F02. 


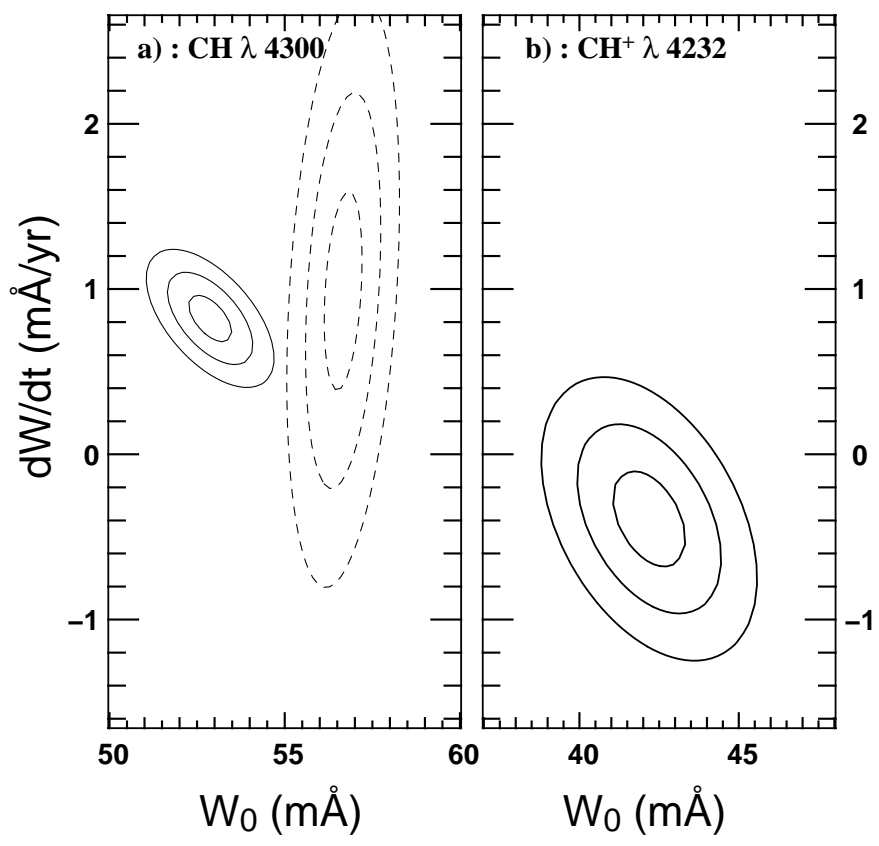

Fig. 4. Linear fit of $W_{\lambda}(t)$ for $\mathrm{CH} \lambda 4300$ a) and $\mathrm{CH}^{+} \lambda 4232$ b) $\left(W_{0}=W_{\lambda}\left(t_{\mathrm{med}}\right)\right)$. Solid line: linear fit to the whole set of data (with $t_{\mathrm{med}}=$ April 1996). Dashed line: linear fit to the recent data $\left(t_{\mathrm{med}}=\right.$ December 2000). The best fits are shown in Fig. 3. Contours represent the levels 1,2 and 3 of the reduced $\left(\chi^{2}-\chi_{\min }^{2}\right)$.

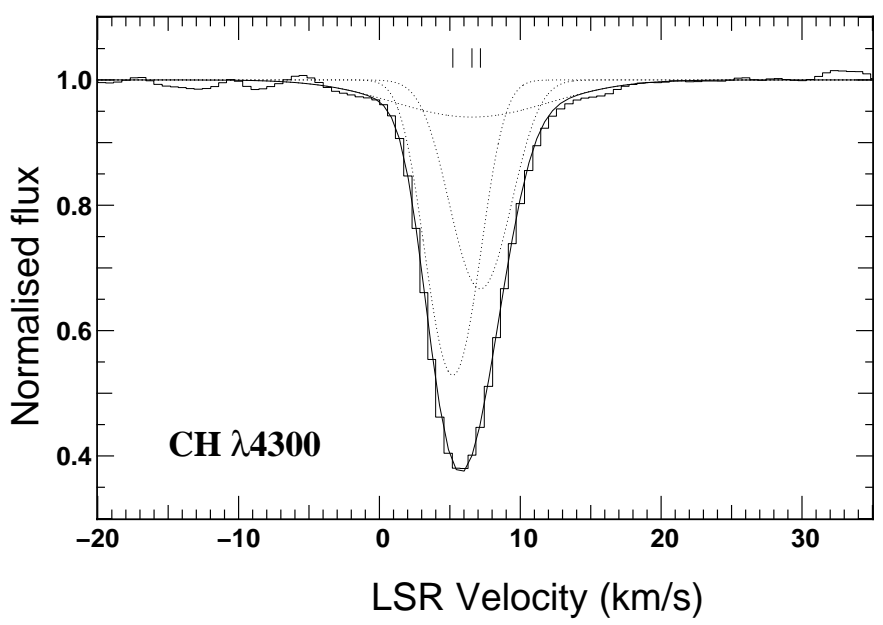

Fig. 5. High resolution observation (F02) of the $\mathrm{CH}$ line. Solid line: fit with VPFIT. Dotted lines: the three components of the fit.

these lines and the $S / N$ attained, we find that even the strongest feature at $3886 \AA$ does not bring useful additional constraints.

\subsubsection{High resolution observations}

We first discuss the highest signal-to-noise ratio observation of $\mathrm{CH}$ 14300, performed in 2002 at McDonald (F02). The profile was fitted using VPFIT, taking into account the doublet structure of the $\mathrm{CH}$ ground level (Lien 1984). Both levels are assumed to have the same column density. Three components are needed: two narrow ones reproduce the slightly asymmetric core of the absorption line and a much broader and fainter one accounts for the wings which are present on both sides of the

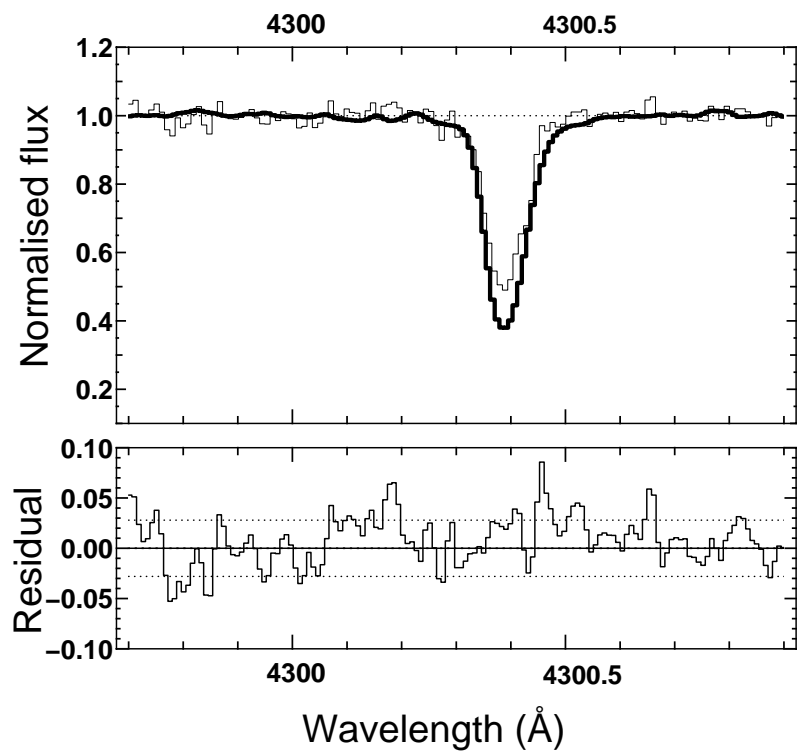

Fig. 6. Variation of the $\mathrm{CH}$ profile. Top panel: F93a and F02 spectra are overplotted as thick and solid line respectively. Bottom panel: the optical depth of F93a is multiplied by 1.34 and the resulting spectrum is subtracted from F02. The residual (solid line) is compared to the $1 \sigma$ noise of F93a (dotted line).

core. The resulting fit and the three components are shown in Fig. 5 and the parameters of the fit are given in Table 1. There is no doubt about the reality of the broad component since it is clearly seen in all spectra with the appropriate $S / N$ ratio (A91, F93a, F02) and for both the 3886 and $4300 \AA$ lines (H98). It is noteworthy that, although barely noticeable, it contains no less than $28 \%$ of the total $\mathrm{CH}$ column density. As a consistency check, we verified that $W_{\lambda}$ values measured for all $\mathrm{CH}$ lines detected in Herbig's spectrum are consistent with the velocity distribution derived from the F02 spectrum. We note, however, that different 3-component solutions for the latter are also acceptable (in particular, one in which the strongest narrow component is the red one). Nevertheless, since the optical depth remains moderate, this degeneracy does not result in larger uncertainties on $N(\mathrm{CH})$. Moreover, very recent observations of the $\mathrm{CO}(2-1)$ emission line at $1.3 \mathrm{~mm}$ towards HD 34078 performed at the $30 \mathrm{~m}$ IRAM telescope give a velocity profile in excellent agreement with that shown in Fig. 5 (E. Roueff \& M. Gerin, private communication).

If we now fit independently the F93a profile, a slightly different solution is obtained. The parameters are less accurate due to the lower signal-to-noise ratio - see Table 1 . The reality of profile variations is difficult to assess from comparison of the F93a and F02 parameters because the decomposition is not unique; we give in Table 1 solutions with the lowest $\chi^{2}$ but relatively different solutions cannot be rejected. We then perform a more direct comparison of profiles and find that multiplication of the F93a optical depth by 1.34 provides an acceptable fit to the F02 profile (Fig. 6; the slightly different spectral resolutions of the two spectra have been taken into account). Therefore, we conclude that the velocity distribution has remained roughly constant between 1993 and 2002 and that the observed line 
Table 1. Relative velocity, Doppler parameter and column densities derived by fitting the high resolution $\mathrm{CH}$ and $\mathrm{CH}^{+}$profiles with VPFIT. Three components are assumed; results obtained with one single component are also given. Solutions with the lowest $\chi^{2}$ are listed but relatively different solutions cannot be rejected. The doublet structure of the $\mathrm{CH}$ ground level has been taken into account: the column density given in this table is the sum for the two levels. The reduced $\chi^{2}$ is always $\sim 1$.

\begin{tabular}{|c|c|c|c|}
\hline & \multicolumn{3}{|c|}{$\mathrm{CH} \lambda 4300$} \\
\hline & $v_{\mathrm{LSR}}\left(\mathrm{km} \mathrm{s}^{-1}\right)$ & $b\left(\mathrm{~km} \mathrm{~s}^{-1}\right)$ & $\log _{10} N$ \\
\hline \multirow{4}{*}{1993} & $5.0_{-0.5}^{+0.5}$ & $2.0_{-0.3}^{+0.4}$ & $13.58_{-0.06}^{+0.06}$ \\
\hline & $6.2_{-0.8}^{+0.8}$ & $2.6_{-0.6}^{+1.5}$ & $13.20_{-0.17}^{+0.12}$ \\
\hline & $5.5_{-2.0}^{+1.3}$ & $5.9_{-2.2}^{+2.5}$ & $13.19_{-0.17}^{+0.12}$ \\
\hline & 5.4 & 3.0 & 13.85 \\
\hline \multirow{6}{*}{2002} & $5.6_{-0.15}^{+0.25}$ & $1.90_{-0.09}^{+0.08}$ & $13.68_{-0.01}^{+0.03}$ \\
\hline & $7.6_{-0.15}^{+0.25}$ & $2.40_{-0.35}^{+0.10}$ & $13.55_{-0.02}^{+0.04}$ \\
\hline & $7.0_{-0.5}^{+0.5}$ & $7.5_{-0.7}^{+0.8}$ & $13.16_{-0.05}^{+0.04}$ \\
\hline & 6.4 & 3.0 & 13.98 \\
\hline & \multicolumn{3}{|c|}{$\mathrm{CH}^{+} \lambda 4232$} \\
\hline & $v_{\mathrm{LSR}}\left(\mathrm{km} \mathrm{s}^{-1}\right)$ & $b\left(\mathrm{~km} \mathrm{~s}^{-1}\right)$ & $\log _{10} N$ \\
\hline \multirow{4}{*}{1993} & $7.5_{-0.2}^{+0.2}$ & $2.5_{-0.2}^{+0.2}$ & $13.58_{-0.02}^{+0.04}$ \\
\hline & $5.5_{-0.2}^{+0.2}$ & $2.7_{-0.4}^{+0.2}$ & $13.41_{-0.03}^{+0.04}$ \\
\hline & - & - & - \\
\hline & 6.7 & 3.0 & 13.80 \\
\hline \multirow{4}{*}{2002} & $7.0_{-0.3}^{+0.3}$ & $2.2_{-0.3}^{+0.2}$ & $13.55_{-0.03}^{+0.04}$ \\
\hline & $4.6_{-0.3}^{+0.3}$ & $2.3_{-0.4}^{+0.4}$ & $13.30_{-0.05}^{+0.05}$ \\
\hline & $7.8_{-2.5}^{+2.5}$ & $10_{-3}^{+4}$ & $13.00_{-0.12}^{+0.10}$ \\
\hline & 6.2 & 3.0 & 13.82 \\
\hline
\end{tabular}

variation is essentially due to an increase by the same factor of the column density for each of the three components.

For the velocity profile obtained above, the ratio $(\delta N / N) /\left(\delta W_{\lambda} / W_{\lambda}\right)$ needed to translate equivalent width variations into $N$ variations increases from 1.37 to 1.50 between $W_{\lambda}=48$ and $W_{\lambda}=60 \mathrm{m \AA}$. For a single component profile, these ratios equal 1.36 and 1.49 respectively, showing that in our optical depth regime, $(\delta N / N) /\left(\delta W_{\lambda} / W_{\lambda}\right)$ depends very weakly on the exact model chosen to fit the profiles. This implies an increase of $\Delta N / N=22 \%$ in either the step function fit or in the linear fit (between 1992 and 2001). In this latter case, bounds of $12 \%<\Delta N / N<38 \%$ are obtained at the $3 \sigma$ level. The higher $(34 \%)$ increase in $N(\mathrm{CH})$ between F93a and F02 corresponds to a $12 \mathrm{~m} \AA$ increase in $W_{\lambda}$.

\subsection{Structure in $\mathrm{CH}^{+}$}

$\mathrm{CH}^{+}$transitions are observed at $3957 \AA$ and $4232 \AA$ in the OHP data; some additional lines are detected at shorter wavelengths in G. Herbig's spectrum. The absorption line at $3957 \AA$ is free of blending and the continuum can be accurately determined. Although the $4232 \AA$ line is more difficult to measure, the $S / N$ ratio in this region is higher and thus comparable accuracy is obtained for the two lines.

\subsubsection{Small scale variations}

Both lines appear to be roughly constant in equivalent width (Figs. 3b, c). Erratic variations still seem to be present, although the larger errors yield an acceptable value of $\chi^{2} \sim 1$ for the best fit. In particular, the pattern characterising the time variations is similar for both $\mathrm{CH}^{+}$lines and for the $\mathrm{CH} \lambda 4300$ transition. This is especially clear for the last four OHP measurements, marked with letters A to D in Fig. 3. We also note that the F02 measurement, which has been made in completely independent conditions, apparently displays this coherent $\mathrm{CH} / \mathrm{CH}^{+}$behavior. Finally, it appears in Figs. $3 \mathrm{a}$ and $3 \mathrm{~b}$ that closely-spaced measurements (made during winter 1999-2000) display relatively smaller scatter, indicating a possible cut-off in the structure at scales below 1-2 AU.

Such a coherent variation could be an artefact due to instrumental effects (e.g., if changes in the background were not taken into account properly). We searched for any dependence on the observing conditions (e.g., high or low sky background) and found none. We also selected a few narrow stellar lines near $4300 \AA$ for which the continuum level is well defined and measured their equivalent width; no significant variations of the kind observed for $\mathrm{CH}$ or $\mathrm{CH}^{+}$are observed. Finally, we checked that the depth of the stellar $\mathrm{H} \gamma$ absorption is stable as it should be in the absence of uncontroled variations of the zero level.

We therefore strongly suspect that stochastic correlated fluctuations of $N(\mathrm{CH})$ and $N\left(\mathrm{CH}^{+}\right)$are present, although more accurate measurements (especially for $\mathrm{CH}^{+}$) would be useful to establish more firmly their reality and to characterise their properties. Among measurements performed after November 1999 , the rms fluctuation of $W_{\lambda}(4300)$ is $1.5 \mathrm{~m} \AA$ while that of $W_{\lambda}(4232)$ is $1.8 \mathrm{~m} \AA$ (the corresponding fluctuations in $N$ are discussed below).

\subsubsection{Large scale variations}

Figure $4 \mathrm{~b}$ shows the result of a linear fit to the whole set of data for $\mathrm{CH}^{+}$14232. The minimum $\chi^{2}$ is again close to 1 . Although the best fit is a slow decrease, $\mathrm{d} W_{\lambda} / \mathrm{d} t=-0.4 \mathrm{~m}^{\circ} \mathrm{yr}^{-1}$, a constant value, $W_{\lambda} \sim 40-43 \mathrm{~m} \AA$, cannot be rejected at the $1.5 \sigma$ level. We obtain the following $3 \sigma$ bounds: $-1.2<\mathrm{d} W_{\lambda} / \mathrm{d} t<$ $0.45 \mathrm{m \AA} \mathrm{yr}^{-1}$. Clearly, the variation observed for $\mathrm{CH}^{+}$is quite different from that seen for $\mathrm{CH}$.

Consistent results are obtained from $\mathrm{CH}^{+} \lambda 3957$. The ratio of the two total $\mathrm{CH}^{+}$equivalent widths, $W_{\lambda}(3957) / W_{\lambda}(4232)$, is found to be constant at a value of $0.65 \pm 0.05$, indicating that these lines are nearly optically thin (ratio of 0.60 ). The best linear fit is also decreasing, although a constant value for both $W_{\lambda}(3957)$ or $W_{\lambda}(4232)$ cannot be ruled out. 


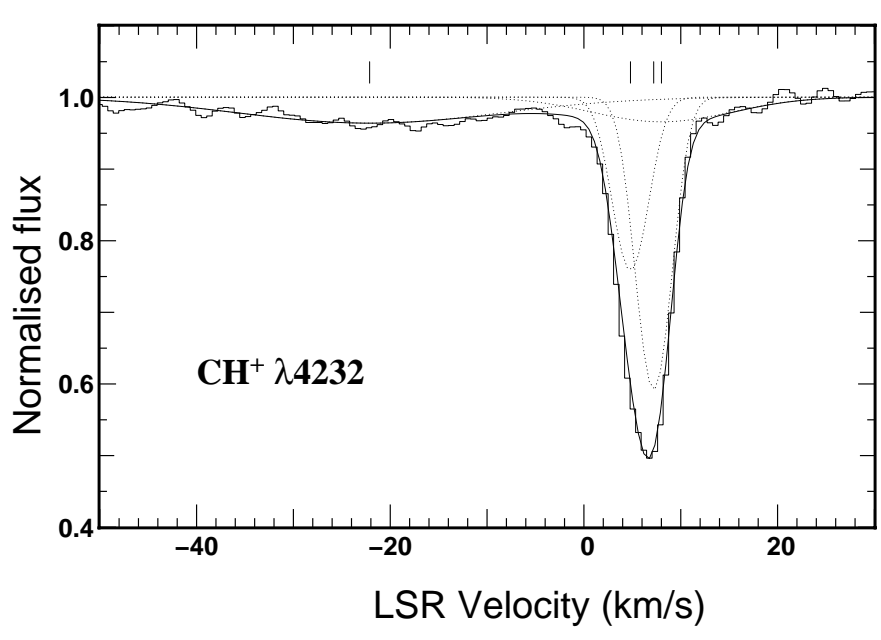

Fig. 7. High resolution observation (F02) of the $\mathrm{CH}^{+} \lambda 4232$ line (dotted lines: the three components of the fit and the stellar line; solid line: resulting fit).

\subsubsection{High resolution observations}

$\mathrm{CH}^{+} \lambda 4232$ has been observed twice at high resolution (F93a and F02). To fit these profiles, we first remove the blended stellar line using a specific Gaussian profile. The $\mathrm{CH}^{+}$line in F02 is asymmetric, as was the $\mathrm{CH} \lambda 4300$ one, but now it is the blue wing which is more extended. A good fit is obtained with 3 components; parameters are summarised in Table 1 and shown in Fig. 7.

The F93a and F02 profiles are almost identical. The same components fit both observations with a residual less than 1.5 and $1.0 \sigma$, respectively. If the F93a spectrum is analysed separately, a good fit is obtained with two narrow components only, but this is just due to the lower signal-to-noise ratio. The third broad component is also apparent in the H98 spectrum for both the $\mathrm{CH}^{+}$lines at 3957 and $4232 \AA$.

In summary, the $\mathrm{CH}^{+}$line profiles have been stable between 1990 and 2002. For the model derived from the F02 profile and the $\mathrm{CH}^{+} \lambda 4232$ data, one gets $\delta N / N=1.3 * \delta W_{\lambda} / W_{\lambda}$ in the range $\delta W_{\lambda}=-10$ to $10 \mathrm{~m} \AA$. We then derive an upper limit for the annual variation of the column density from 1990 to 2002 of $-3.9<\delta N / N<1.45 \%$ per year at the $3 \sigma$ level. Tighter constraints can be obtained by combining $\mathrm{CH}^{+} \lambda 3957$ and $\mathrm{CH}^{+} \lambda 4232$ measurements. For all epochs, we derive $N\left(\mathrm{CH}^{+}\right)$and $\sigma(N)$ from each transition; estimates drawn from $\mathrm{CH}^{+} \lambda 4232$ appear to be, in average, slightly lower than those inferred from $\mathrm{CH}^{+} \lambda 3957$, presumably because the blend with the stellar feature induces an underestimate of $W_{\lambda}(4232)$. We thus apply a small positive and constant offset to $W_{\lambda}(4232)$ measurements in order to get the same $<N>$ for both transitions and finally compute the $\left(\sigma(N)^{-2}\right.$ weighted) average for each epoch. The best fit corresponds to $\delta N / N=-0.65 \%$ per year and the $3 \sigma$ bounds are -1.5 and $0.27 \%$ per year (a non varying $N\left(\mathrm{CH}^{+}\right.$) is rejected at the $2 \sigma$ level); these values clearly exclude an increase as large as that seen for $\mathrm{CH}$.

Let us now compare the velocity profiles of $\mathrm{CH}$ and $\mathrm{CH}^{+}$absorptions. Figure 8 shows the apparent optical depth versus velocity for both species. It can be seen that no

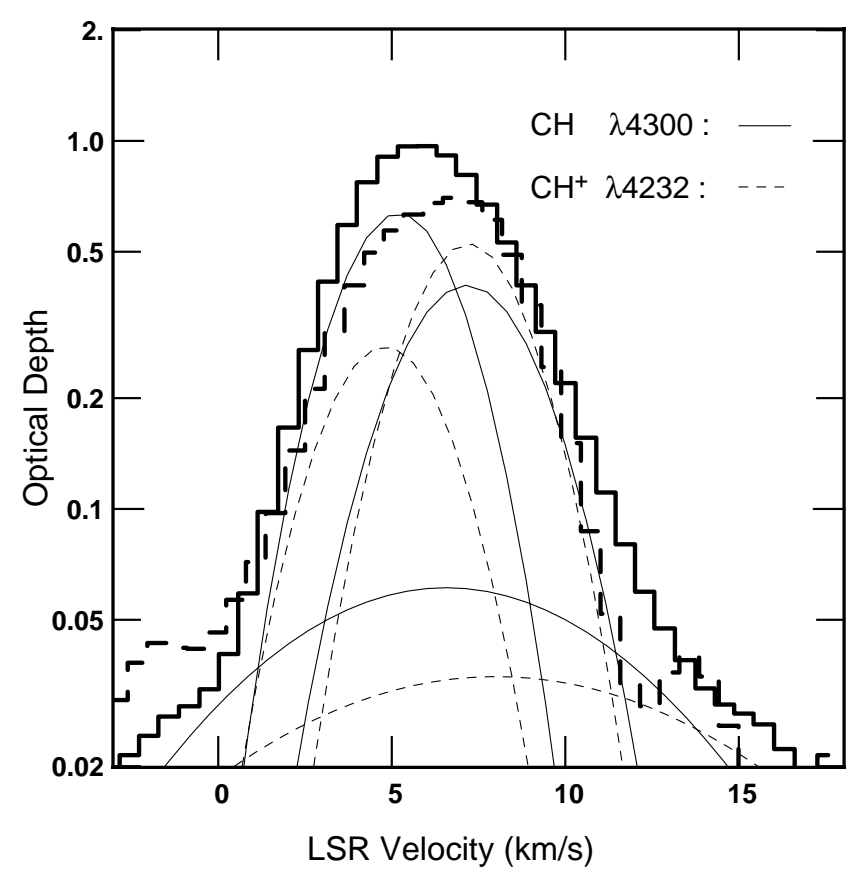

Fig. 8. Comparison of optical depth profiles for high resolution observations (F02) of $\mathrm{CH} \lambda 4300$ (solid line) and $\mathrm{CH}^{+} \lambda 4232$ (dashed line). Thick lines correspond to observed profiles while thin ones represent individual components.

systematic shift is present; observed profiles are quite similar although the asymmetry of $\mathrm{CH}$ and $\mathrm{CH}^{+}$lines is reversed. This is clearly seen when comparing the two narrow components needed to fit the profiles. (A comparison of the broad components would be meaningless because the $\mathrm{CH}^{+}$one is ill-defined due to blending with the adjacent stellar absorption.) The $\mathrm{CH}$ and $\mathrm{CH}^{+}$components have the same velocities but different $\mathrm{CH}^{+} / \mathrm{CH}$ ratios, as often observed (see e.g., Crawford 1989 and the $\xi$ Per profiles given by Crane et al. 1995).

\subsection{Structure in $\mathrm{CN}$}

Because $\mathrm{CN}$ is expected to respond strongly to density changes, the behavior of this species can give useful clues concerning the nature of the $\mathrm{CH}$ variations (Pan et al. 2001). The available observations were taken in 1993 (F93b), 1998 (H98) and 2002 (F02). Unfortunately, when seen towards hot stars, the $R(0)$ line is blended with stellar features from O II and C II (Fig. 9; see Meyer \& Jura 1985). As a result of the possibility of blending, we remeasured the line in a consistent manner. In practice, this is not easy because the resolution differs from one spectrum to another and further, the broad stellar feature appears to vary in time. Results are given in Table A.2; the larger value quoted by Herbig (1999) for the $R(0)$ line is likely due to inclusion of the stellar feature.

\subsubsection{Analysis of G. Herbig's spectrum}

Since the Keck data have the highest $S / N$ ratio, we first discuss this spectrum and its implication on the $\mathrm{CN}$ column density, velocity distribution and excitation. The $R(0), R(1)$ and $P(1)$ lines are clearly detected, as shown in Fig. 9. 


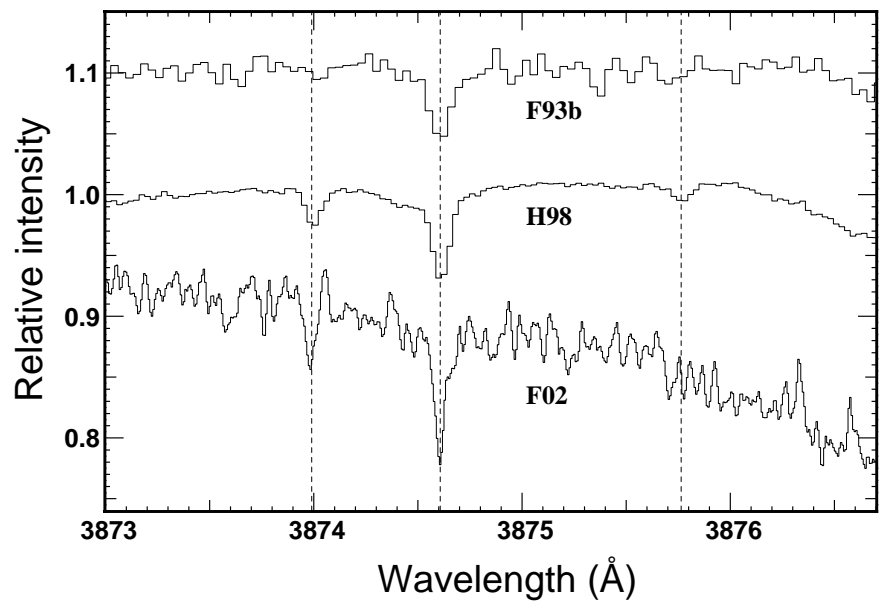

Fig. 9. The $\mathrm{CN}(0,0)$ lines in the F93b, H98 and F02 spectra. In the H98 spectrum, the $S / N$ ratio is 700 per $0.07 \AA$ pixel. $R(0)$ lines have been aligned at the rest wavelength. The three spectra have been normalised to 1 at $\lambda \sim 3875$ and shifted in y for clarity. Note the broad stellar feature on the blue side of the $R(0)$ lines.

To our knowledge, there exist no high resolution observation with adequate $S / N$ of the $\mathrm{CN}$ lines towards HD 34078 that would allow us to measure their optical depth and investigate saturation effects in a direct way. Classically, the latter are estimated by comparing the relative strengths of the $R(1)$ and $P(1)$ lines (Meyer \& Jura 1985). However, these $\mathrm{CN}$ absorptions are quite weak in the HD 34078 spectrum $\left(W_{\lambda}=2.6 \pm 0.1 \mathrm{~m} \AA\right.$ and $W_{\lambda}=1.5 \pm 0.1 \mathrm{~m} \AA$ respectively, while for the $R(0)$ line, $W_{\lambda}=$ $5.7 \pm 0.2 \mathrm{~mA}$ ), and the equivalent width ratio poorly constrains the $b$ value. Indeed, only values smaller than $0.1 \mathrm{~km} \mathrm{~s}^{-1}$ can be rejected (at the $2 \sigma$ level); thus, within errors, the equivalent width ratio is consistent with $\mathrm{CN}$ lines being optically thin.

Another way to estimate the optical depth of the $3874.6 \AA$ $R(0)$ line is to compare its equivalent width to that of the $R(0)$ line from the $(1,0)$ vibrational band at $3579.96 \AA$ (Meyer et al. 1989). In Herbig's spectrum, a weak feature is present at the expected position with $W_{\lambda}=0.55 \pm 0.15 \mathrm{m \AA}$; in the optically thin limit, a value $W_{\lambda}=0.50 \pm 0.02 \mathrm{~m} \AA$ would be expected. Again, within errors, the relative strengths of the two $R(0)$ features are consistent with the assumption that these two lines are thin and only a lower limit on $b$ can be obtained: $b>0.2 \mathrm{~km} \mathrm{~s}^{-1}(2 \sigma$ limit).

In the optically thin limit, the observed $W_{\lambda}$ ratio for $R(0)$ and $R(1)$ implies an excitation temperature $T_{\text {exc }}=3.6 \pm 0.2 \mathrm{~K}$ (following the same method as Meyer \& Jura 1985), significantly above the value expected $T_{\text {exc }}=T_{\mathrm{CMB}}=2.73 \mathrm{~K}$ if the excitation were due only to interaction with $\mathrm{CMB}$ radiation. As discussed above, the available data yield only loose constraints on $b(\mathrm{CN})$ and if the latter were small enough, saturation effects might affect the determination of $T_{\text {exc }}$. To assess the importance of these effects, we compute $T_{\text {exc }}$ assuming $b=1,0.5,0.25$, $0.20 \mathrm{~km} \mathrm{~s}^{-1}$ and get $T_{\text {exc }}=3.5,3.4,3.0$ and $2.8 \mathrm{~K}$ respectively. For lines of sight with similar extinction, $b(\mathrm{CN})$ is observed to be about $1 \mathrm{~km} \mathrm{~s}^{-1}$ and values as low as $0.2 \mathrm{~km} \mathrm{~s}^{-1}$ appear unlikely in comparison with available determinations (Crawford 1995). Such low values for $b$ would also be at odds with the results for $\mathrm{CH} \lambda 4300$; no component in $\mathrm{CH}$, that is associated with $\mathrm{CN}$, has such a low $b$-value. We conclude that $T_{\text {exc }}$ very likely exceeds $T_{\mathrm{CMB}}$, implying that collisions with electrons contribute significantly in populating the $N=1$ level (Black \& van Dishoeck 1991). If $b(\mathrm{CN})=1 \mathrm{~km} \mathrm{~s}^{-1}, T_{\text {exc }}$ would be among the highest values quoted by Black \& van Dishoeck (1991).

\subsubsection{McDonald 2002 and 1993 spectra}

Very recently, the $\mathrm{CN}(0,0)$ lines have been reobserved at McDonald ( $R=170$ 000) (Fig. 9). The stellar feature around the $R(0)$ line is not well defined due to limited signal to noise; apparently, it is weaker than in Herbig's spectrum. The $R(0)$ and $R(1)$ lines are clearly detected with $W_{\lambda}=6.3 \pm 0.5 \mathrm{m \AA}$ and $W_{\lambda}=2.6 \pm 0.4 \mathrm{~m} \AA$ respectively. These values are compatible with the 1998 ones. On the other hand, the 1993 (F93b) spectrum yields $W_{\lambda}=5.1 \pm 0.2 \mathrm{~m} \AA$ and $W_{\lambda}<2.0 \mathrm{m \AA}$ ( $2 \sigma$ limit) for the $R(0)$ and $R(1)$ lines, implying $T_{\text {exc }}<3.1 \mathrm{~K}$. Altogether, the data suggest that both the $N=0$ and $N=$ $1 \mathrm{CN}$ column densities have increased, even if the variation is only marginally significant $(\Delta N / N=12 \pm 6 \%)$. Therefore, the $\mathrm{CN}$ variation could be as high as that for $\mathrm{CH}$, but not much larger.

\subsection{Structure in DIBs carriers}

The OHP spectra acquired for our study of $\mathrm{CH}$ and $\mathrm{CH}^{+}$contain several strong DIBs. We now use these data together with older spectra to investigate the spatial distribution of DIBs carriers. Some of them are known to behave differently with respect to the abundance of CH (Krelowski et al. 1999). Then, an immediate goal is to determine whether the kind of small and large scale variations seen for $\mathrm{CH}$ are also present for DIBs.

Among all detected DIBs, we initially selected those at 5780, 5797, 6196, 6284 and $6614 \AA$ which are relatively strong (opacity reaching 6\%) and narrow. For broader ones, the continuum level and shape is difficult to determine and only large variations could be seen. Among the five features quoted above, we find that the sensitivity needed to detect variations with an amplitude comparable to that of the observed $\mathrm{CH}$ changes can be obtained only for the 5780 and $5797 \AA$ features. We then focus on these two DIBs. Since they are fully resolved (except possibly for some substructure), we can perform a direct comparison of the absorption profiles, once the spectra have been brought to a common wavelength scale. Equivalent width measurements would not be appropriate due to large continuum placement errors.

We first discuss variations at the scale of $0.5-1$ year ( $\simeq 10 \mathrm{AU})$ among the spectra taken at OHP. Since the latter have been taken with the same instrument, the shape of the continuum adjacent to the DIBs considered is very stable, rendering the intercomparison much easier. We selected pairs of spectra (October 2000/March 2002 and February 2001/October 2001) in which $W_{\lambda}(4300)$ measurements show the largest difference (about $5.3 \%$ which corresponds to relative variations in $N(\mathrm{CH})$ of $7.2 \%$ ). No significant variation is detected at the level of 10 and $15 \%$ for the 5780 and $5797 \AA$ DIBs, respectively. To derive these upper limits, we artificially increase/decrease the strength 

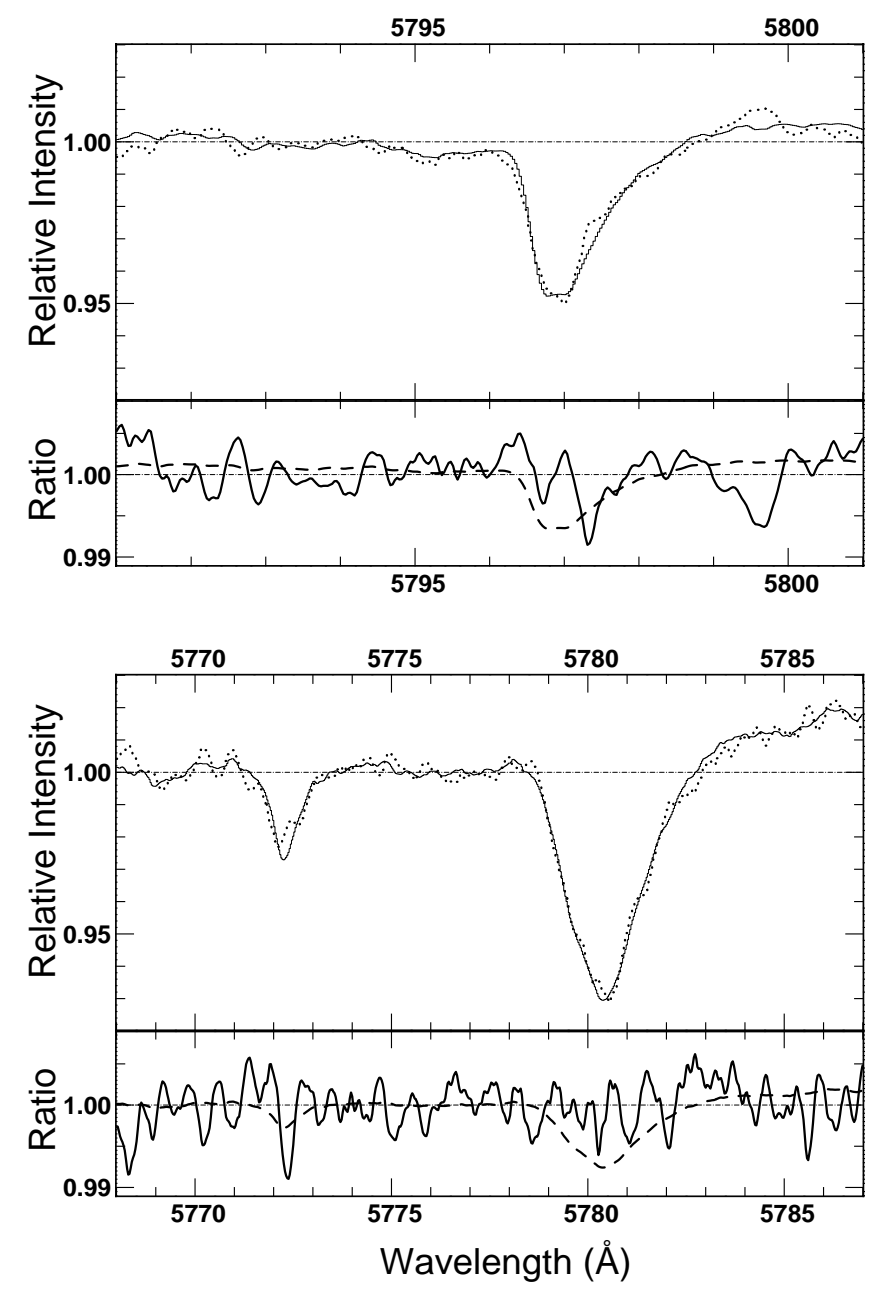

Fig. 10. Time variation of the $5780 \AA$ and $5797 \AA$ DIBs. Upper panels: the average of all OHP spectra (solid line) is compared to the spectrum obtained by Jenniskens et al. (1992) (dotted line). Lower panels: the ratio of the OHP and J92 spectra (solid line) is compared to the ratio obtained after artificially increasing by $10 \%$ and $15 \%$ the respective strengths of the 5780 and $5797 \AA$ DIBs in the OHP spectrum (dashed line).

of the DIB considered in the epoch 1 spectrum and then compare the latter to epoch 2 data. Then, the sensitivity reached is not large enough to detect variations as faint as those displayed by $N(\mathrm{CH})$ over a period of about one year.

To study variations at larger scales, we use the spectrum obtained by Jenniskens et al. (1992) and compare it to the average of all our OHP spectra. The ratio of the two smoothed spectra is computed, and to ensure a good "relative normalisation", we fit values taken by this ratio in a few windows adjacent to each DIB with a polynomial of order 1 or 2 . We then divide the first spectrum by this relative normalisation. The spectra thus obtained are shown in Fig. 10 and show no variation of the 5780 and $5797 \AA$ DIBs at respective levels of 10 and $15 \%$. Since both DIBs are optically thin, these upper limits are to be compared to the relative variation in $N(\mathrm{CH})$ which is estimated to be $20 \%$ over the same period. We then conclude that the $5780 \AA$ DIB at least has varied less than $\mathrm{CH}$.

\subsection{Summary of observational results}

The main results that emerge from observations are the following:

- Over the past 12 years (or scales of 100-200 AU in the foreground cloud), the $\mathrm{CH}$ column density has increased by $12-38 \%$. A linear form (with $\mathrm{d} N /(N \mathrm{~d} t)=1.4 \% \mathrm{yr}^{-1}$ ) or a step function $(\triangle N / N=22 \%$ in 1998) can both fit this overall variation. Over the same period, the $\mathrm{CH}^{+}$column density displays a markedly different behavior (a slow decrease with $\mathrm{d} N /(N \mathrm{~d} t)=$ $\left.-0.65 \% \mathrm{yr}^{-1}\right)$.

- On the scale of a few months ( $\sim 5 \mathrm{AU})$, correlated fluctuations in $N(\mathrm{CH})$ and $N\left(\mathrm{CH}^{+}\right)$are strongly suggested by the data. A rough estimate of their amplitude over the period November 99-March 02 can be inferred from the rms scatter of $W_{\lambda}$ measurements: $\sigma(N(\mathrm{CH})) / N(\mathrm{CH}) \approx 3.6 \%$ and $\sigma\left(N\left(\mathrm{CH}^{+}\right)\right) / N\left(\mathrm{CH}^{+}\right) \approx 5.8 \%$.

- $\mathrm{CH}$ and $\mathrm{CH}^{+}$velocity profiles show no significant time variation.

- CN observations indicate a moderate increase of $N(\mathrm{CN})$ but a higher excitation temperature in 1998 and 2002, as compared to 1993 . The recent $T_{\text {exc }}$ value is significantly above that expected from excitation by CMB radiation alone.

- The 5780 and $5797 \AA$ DIBs have been stable and the former has varied by no more than half that seen in $\mathrm{CH}$.

\subsection{Discussion}

\subsubsection{AU scale structure and $\mathrm{CH}^{+}$production}

We now discuss structure in the gas containing $\mathrm{CH}$ and $\mathrm{CH}^{+}$ at the 1-10 AU scale as probed by the homogeneous observations performed since 1999 . We found indications for fluctuations of $\mathrm{CH}$ and $\mathrm{CH}^{+}$absorptions: first, the scatter is relatively large and second, $\mathrm{CH}$ and $\mathrm{CH}^{+}$fluctuations are apparently correlated. Nevertheless, it should be stressed that these variations are quite small. Indeed, part of the observed scatter can be attributed to measurement errors and the real scatter in $N(\mathrm{CH})$ or $N\left(\mathrm{CH}^{+}\right)$cannot exceed a few $\%$. This is much smaller than the $\mathrm{H}_{2} \mathrm{CO}$ variations described by Moore \& Marscher (1995), which attain $17 \%$ and smaller than $N(\mathrm{H}$ I) fluctuations seen in the atomic phase over similar scales. In a simple model where most of the $\mathrm{CH}$ or $\mathrm{CH}^{+}$gas is comprised of identical discrete entities, the number of such "clumps" must exceed $10^{3}$ to get fluctuations that weak.

If the erratic variations seen since 1999 reflect the geometry of localised regions where $\mathrm{CH}^{+}$molecules are produced, then it is natural to detect associated variations of $\mathrm{CH}$ since about $30 \%$ of the $\mathrm{CH}$ molecules in a given sight line form from $\mathrm{CH}^{+}$but the fraction approaches $100 \%$ for gas with densities less than about $100 \mathrm{~cm}^{-3}$ (Gredel et al. 2002). Presently, it is difficult to check the consistency of this picture because the relative amplitudes of the $\mathrm{CH}$ and $\mathrm{CH}^{+}$fluctuations are poorly constrained by the available data. Alternatively, in a picture where $\mathrm{CH}^{+}$ is produced throughout the low density medium (e.g., Draine \& Katz 1986; Pineau des Forêts et al. 1986; Spaans 1995; Federman et al. 1996), the correlation between $\mathrm{CH}$ and $\mathrm{CH}^{+}$ could be due to a physical mechanism which induces local 
density enhancements for all species. For instance, compression in regions where chaotic flows happen to converge has been invoked to account for the AU-scale structure in atomic gas (Jenkins \& Tripp 2001; Hennebelle \& Pérault 1999) and could also be effective in molecular gas. If the lifetime of these transient fragments is small enough, such a process would lead to comparable values for $\delta N / N(\mathrm{CH})$ and $\delta N / N\left(\mathrm{CH}^{+}\right)$; otherwise, the higher density might result in efficient $\mathrm{CH}^{+}$destruction through reactions with $\mathrm{H}_{2}$ and rather produce an anticorrelation. One way to disentangle the above two scenarios in which small scale structure is either related to chemically active regions or just to pressure fluctuations would be to study the behavior of other species whose chemistry is not directly linked to that of $\mathrm{CH}$ and $\mathrm{CH}^{+}$.

\subsubsection{The long-term $\mathrm{CH}$ variation: Evidence for a dense fragment?}

Over larger scales (100-200 AU), $\mathrm{CH}$ and $\mathrm{CH}^{+}$clearly display distinct behaviors. Given the number of independent measurements and consistency among results, we consider that this difference is well established, even if the form of the $\mathrm{CH}$ increase is not well understood. In particular, we cannot exclude an abrupt variation by $20 \%$ occurring in 1997-1998. The corresponding $\mathrm{CN}$ absorption has increased only modestly $(\delta N / N=$ $12 \pm 6 \%$ ) between 1993 and 1998, but the data strongly suggest a change in excitation from $T_{\text {exc }}<3.1 \mathrm{~K}$ in 1993 to $T_{\text {exc }}=3.6 \pm$ $0.17 \mathrm{~K}$ in 1998 . The 1998 value is quite high in comparison with results quoted by Black \& van Dishoeck (1991) for other lines of sight, implying a high electron density. The fact that the ratio $N(\mathrm{CN}) / N(\mathrm{CH})$ has remained roughly constant while $N(\mathrm{CH})$ and $N(\mathrm{CN})$ have increased, indicates that little change in gas density has accompanied the fluctuations in columns (see Pan et al. 2001).

Consider a model in which the increase in $N(\mathrm{CH})$ by about $20 \%$ over a time interval of 1 to 10 years is due to a newly intervening clump. $N(\mathrm{CH})$ is known to vary linearly with $N\left(\mathrm{H}_{2}\right)$ with a ratio $N(\mathrm{CH}) / N\left(\mathrm{H}_{2}\right) \approx 4.0 \times 10^{-8}$ (Federman 1982; Roueff 2001), Towards HD 34078, a value larger by a factor of four is inferred (see Fig. 2 from Roueff 2001). Assuming that such a ratio is still valid at the scales considered here, one can easily compute the associated increase in $N\left(\mathrm{H}_{2}\right)$ : $1.1 \times 10^{20} \mathrm{~cm}^{-2}$. Assuming an intermediate value of $50 \mathrm{AU}$ for the transverse scale $[N(\mathrm{CH})$ has remained at its "high" value at least for 4 years] and a comparable size along the line of sight, the implied density for that hypothetical fragment is around $1.5 \times 10^{5} \mathrm{~cm}^{-3}$. In such a dense fragment, very little $\mathrm{CH}^{+}$is expected to be present due to reactions with $\mathrm{H}_{2}$, accounting for the absence of an associated increase in $N\left(\mathrm{CH}^{+}\right)$. Similarly, the stability of the 5780 and $5797 \AA$ DIBs can be understood in this scenario since DIBs carriers are known to lie preferentially in diffuse media. However, the $\mathrm{CN} / \mathrm{CH}$ ratio is expected to be large at these densities and the relative increase of $N(\mathrm{CN})$ should be much larger than that of $\mathrm{CH}$, contrary to what is observed. The density quoted above could be lowered if the structure were elongated along the line of sight (cf. the model proposed by Heiles (1997) for atomic gas), but a very large (and unrealistic!) aspect ratio would be needed to bring it to values typical for that kind of material (a few times $\left.10^{2} \mathrm{~cm}^{-3}\right)$. In particular, Federman et al. (1994) estimated $n_{\text {tot }} \approx$ $200 \mathrm{~cm}^{-3}$ from $N(\mathrm{CN}) / N(\mathrm{CH})$ and $\mathrm{C}_{2}$ observations, at a time when $N(\mathrm{CH})$ had its low value, but the column density ratio has not changed appreciably since then. While it is not clear which $\mathrm{CH}$ component contains $\mathrm{CN}$ as well, the current version of the chemical model (Knauth et al. 2001; Pan et al. 2001) gives similar densities.

Another problem with this model (in addition to understanding how the large overpressure inside the clump can be generated and maintained long enough) is related to the change in the excitation of $\mathrm{CN}$ suggested by the data. Since the variation in $N(\mathrm{CN})$ has been quite limited (at most 20 to 30\%), the higher $T_{\text {exc }}$ value estimated for the whole gas in 1998 and 2002 implies an even larger $T_{\text {exc }}$ in the clump if the rest of the gas has remained at $T_{\mathrm{exc}} \approx 2.7 \mathrm{~K}$. Again, this points towards a large clump density or electron fraction.

A last difficulty involves the observed velocity distribution. The presence of an additional fragment on the 2002 line of sight should have resulted in a new velocity component, but the whole distribution (in particular the two narrow components) has varied globally, without any noticeable change in shape. One could imagine that the additional component is very narrow and does not show up at $R=200000$, but such a feature would be atypical with respect to profiles observed at very high resolution.

\subsubsection{Contribution from gas located near HD 34078 ?}

The difficulties encountered above when attempting to account for the $\mathrm{CH}-\mathrm{CN}$ variations might be related to several peculiarities of the HD 34078 line of sight: i) the large amount of highly excited $\mathrm{H}_{2}$, ii) the large $\mathrm{CH} / \mathrm{H}_{2}$ and $\mathrm{CH}^{+} / \mathrm{H}_{2}$ ratios (note however that the $\mathrm{CH} / \mathrm{CH}^{+}$ratio is in good agreement with the $\mathrm{CH}-\mathrm{CH}^{+}$correlation observed by Gredel 1997), and iii) the large $T_{\text {exc }}$ value for CN. Since point i) is attributed to the influence of the UV bright star upon nearby material (Le Petit et al. 2001; Boissé et al. 2002), one might wonder whether the properties observed for $\mathrm{CH}, \mathrm{CH}^{+}$and $\mathrm{CN}$ could also be the result of this interaction. In this model, $\mathrm{CH}, \mathrm{CH}^{+}$and $\mathrm{CN}$ are expected to be largely photodissociated in the PDR where $\mathrm{H}_{2}$ is excited to high energies.

Then, to account for the observed $\mathrm{CH}$ and $\mathrm{CH}^{+}$amounts, one may imagine that $\mathrm{CH}^{+}$production occurs in the PDR itself, as has been suggested for the Pleiades (White 1984) and IC 348 (Snow 1993). In this scenario, $\mathrm{CH}^{+}$is the result of reactions between $\mathrm{C}^{+}$and $\mathrm{H}_{2}$ in gas at elevated temperatures caused by enhanced $\mathrm{H}_{2}$ photodissociation. The large amount of $\mathrm{CH}$ then arises from the synthesis of $\mathrm{CH}^{+}$and the increase in $T_{\text {exc }}$ occurs because there is enhanced ionization produced by the star's UV radiation. One potential difficulty involves the velocity distribution which appears to be quite narrow (except for the broad component) and stable 
(cf. Herbig 1999). This suggests quiescent absorbing material rather than gas located near a bow shock around a O9 star moving at more than $100 \mathrm{~km} \mathrm{~s}^{-1}$. Possibly, we are seeing "composite absorption" from both pieces of gas, one located close to HD 34078 and strongly influenced by it, and the other consisting of more distant material where the radiation field is enhanced by only a factor of a few relative to the average galactic field.

\subsubsection{Prospects}

Let us now suggest a few prospects concerning additional observations that might clarify the situation. First, we wish to stress the importance of data other than those considered in this paper that could have been taken before 1999, especially spectra obtained in 1997-1998, which would be very useful in constraining the shape of the long-term variation in $\mathrm{CH}$. We thus prompt any reader who would have such data in hand to contact us. Evidently, it is important to continue the $\mathrm{CH}$ and $\mathrm{CH}^{+}$observations with increased $S / N$ ratio in order to characterise better the properties of erratic fluctuations on timescales of about one year. At the same time, these data would give information on the shape of the long-term variation of $\mathrm{CH}$ and $\mathrm{CH}^{+}$absorptions.

To date, FUSE observations have provided 4 spectra, all taken after 1999; their analysis indicates that $N\left(\mathrm{H}_{2}\right)$ variations - if any - are smaller than about $5 \%$ (Boissé et al. 2002). If $N(\mathrm{CH})$ keeps increasing, we shall be able to verify whether the $\mathrm{CH}-\mathrm{H}_{2}$ correlation still holds at the 10-100 AU scales since the expected increase in $N\left(\mathrm{H}_{2}\right)$ will exceed our detection limit.
The behavior of other species like C I and CO can be investigated also using these spectra. However, this can be done with better sensitivity with HST thanks to higher $S / N$ and spectral resolution. HST/STIS spectra would provide invaluable information on the density via $\mathrm{C}$ I fine structure transitions and $\mathrm{C}_{2}$ lines and allow us to follow with improved accuracy the small and large scale variations of many species (C I, H I, excited $\mathrm{H}_{2}, \mathrm{CO}, \mathrm{C}_{2} \ldots$ ). Repeating the $\mathrm{C}_{2}$ visible observations reported by Federman et al. (1994) could also give direct evidence for the high clump density quoted above.

Acknowledgements. We thank the astronomers and staff who obtained the OHP spectra, M. Allen, F.X. Désert, G. Herbig, J. Krelowski, G. Galazutdinov and F. Musaev who kindly provided their data, and E. Roueff and M. Gerin for obtaining an IRAM CO spectrum. We acknowledge fruitful discussions with J. Black, P. Hennebelle and G. Pineau des Forêts and thank S. Thoraval for his initial participation in this program. S.R.F. was supported in part by NASA LTSA grant NAG5-4957.

\section{Appendix A: Tables}

Table A.1. List of observations. ${ }^{a}$ Resolution near $\mathrm{CH} \lambda 4300 .{ }^{b}$ Interstellar lines that have been included in this study. $\mathrm{CH}$ stands for $\mathrm{CH} \lambda 4300$; $\mathrm{CH}^{+}$for $\mathrm{CH}^{+} \lambda 3957$ and $\mathrm{CH}^{+}$$\lambda 4232$, unless specified; $\mathrm{CN}$ for $\mathrm{CN} \lambda 3875$ (Table A.2). DIBs stands for diffuse interstellar bands (see text for details).

\begin{tabular}{|c|c|c|c|c|c|c|}
\hline Date & Observatory & $S / N$ & $R^{a}$ & Transition $^{b}$ & References & \\
\hline $9-89->1-91$ & $\overline{\mathrm{KPNO}^{c}}$ & 135 & 85000 & $\mathrm{CH}, \mathrm{CH}^{+}$ & Allen (1994) & A91 \\
\hline $06-12 / 1991$ & OHP (Aurélie) & $>100$ & 14300 & $\mathrm{CH}, \mathrm{CH}^{+}(4232), \mathrm{DIBs}$ & Jenniskens et al. (1992) & J91 \\
\hline $11-14-97$ & Terskol & $50-80$ & 45000 & $\mathrm{CH}, \mathrm{CH}^{+}$ & Krelowski et al. (1999) & K97a \\
\hline $11-19-97$ & Terskol & $50-80$ & 45000 & $\mathrm{CH}, \mathrm{CH}^{+}$ & Krelowski et al. (1999) & K97b \\
\hline $01-93$ & McDonald echelle & $45-65$ & 200000 & $\mathrm{CH}, \mathrm{CH}^{+}(4232)$ & & F93a \\
\hline $12-93$ & McDonald Sandiford & $100-150$ & 60000 & $\mathrm{CH}, \mathrm{CH}^{+}, \mathrm{CN}$ & & F93b \\
\hline $02-02$ & McDonald echelle & $100-150$ & 170000 & $\mathrm{CH}, \mathrm{CH}^{+}(4232), \mathrm{CN}$ & & F02 \\
\hline $10-98$ & Keck & 600 & 45000 & $\mathrm{CH}, \mathrm{CH}^{+}, \mathrm{CN}$ & Herbig (1999) & H98 \\
\hline $11-18-99$ & & $135-140$ & & & & $\overline{\text { R99a }}$ \\
\hline $12-22-99$ & & $310-360$ & & & & R99b \\
\hline $1-27-00$ & & $120-200$ & & & & R00a \\
\hline $2-27-00$ & OHP (Elodie) & $105-115$ & 32000 & $\mathrm{CH}, \mathrm{CH}^{+}, \mathrm{DIBs}$ & & Roob \\
\hline $3-18-00$ & & $125-150$ & & & & R00c \\
\hline $10-01-00$ & & $120-155$ & & & & R00d \\
\hline $2-10-01$ & & $105-145$ & & & & R01a \\
\hline $10-2-01$ & & $105-145$ & & & & R01b \\
\hline $3-4-02$ & & $150-160$ & & & & R02 \\
\hline
\end{tabular}


Table A.2. Equivalent width of interstellar lines (all errors are $1 \sigma$ error). ${ }^{a}$ Includes broad absorption (cannot recover the broad component). ${ }^{b}$ Does not include broad absorption from stellar line (visible in all spectra). ${ }^{c} 2 \sigma$ upper limit.

\begin{tabular}{clllll}
\hline \hline & $\mathrm{CH}^{a}$ & $\mathrm{CH}+$ & $\mathrm{CH}^{b}$ & $\mathrm{CN}^{b}$ & $\mathrm{CN}^{b}$ \\
& $4300 \AA$ & $3957 \AA$ & $4232 \AA$ & $R(0)$ & $R(1)$ \\
\hline A91 & $48.3^{0.6}$ & $29.8^{1.4}$ & $44.4^{2.0}$ & $\ldots$ & $\ldots$ \\
J91 & $42.0^{10.0}$ & $\ldots$ & $52.0^{10.0} b$ & $\ldots$ & $\ldots$ \\
F93a & $48.0^{2.0}$ & $\ldots$ & $44.0^{1.7}$ & $\ldots$ & $\ldots$ \\
F93b & $52.0^{1.2}$ & $29.3^{0.8}$ & $43.1^{0.9}$ & $5.1^{0.2}$ & $\leq 2 .{ }^{c}$ \\
K97a & $49.6^{1.0}$ & $28.7^{1.5}$ & $39.8^{3.0}$ & $\ldots$ & $\ldots$ \\
K97b & $52.6^{2.0}$ & $25.4^{2.0}$ & $44.0^{3.0}$ & $\ldots$ & $\ldots$ \\
H98 & $58.0^{0.7}$ & $27.3^{0.2}$ & $42.0^{2.0}$ & $5.7^{0.2}$ & $2.6^{0.1}$ \\
R99a & $55.7^{0.7}$ & $29.0^{2.2}$ & $38.0^{2.0}$ & & \\
R99b & $55.4^{0.5}$ & $27.9^{1.4}$ & $41.5^{2.0}$ & & \\
R00a & $55.2^{0.7}$ & $26.3^{1.5}$ & $40.3^{2.0}$ & & \\
R00b & $57.8^{1.2}$ & $22.8^{2.8}$ & $40.5^{3.0}$ & & \\
R00c & $55.8^{1.0}$ & $24.7^{2.3}$ & $40.0^{2.0}$ & & \\
R00d & $56.6^{1.1}$ & $23.2^{1.9}$ & $39.0^{2.0}$ & & \\
R01a & $58.8^{0.8}$ & $31.9^{2.3}$ & $44.0^{3.0}$ & & \\
R01b & $55.4^{0.7}$ & $24.4^{1.3}$ & $39.0^{3.0}$ & & \\
F02 & $60.0^{1.0}$ & $\ldots$ & $44.0^{2.0}$ & $6.3^{0.2}$ & $2.6^{0.4}$ \\
R02 & $58.1^{1.0}$ & $25.3^{1.6}$ & $42.0^{2.0}$ & & \\
\hline
\end{tabular}

\section{References}

Adams, W. S. 1949, ApJ, 109, 354

Allen, M. M. 1994, ApJ, 424, 754

Baranne, A., Queloz, D., Mayor, M., et al. 1996, A\&AS, 119, 373

Black, J. H., \& van Dishoeck, E. F. 1991, ApJ, 369, L9

Boissé, P., Rollinde, E., Le Petit, F., et al. 2001, Proceedings of the XVII IAP Colloquium Gaseous Matter in Galaxies and Intergalactic Space, 51

Boissé, P., et al. 2002, A\&A, submitted

Brown, A. G. A., Hartmann, D., \& Burton, W. B. 1995, A\&A, 300, 903

Carswell, R. F., Webb, J. K., Baldwin, J. A., \& Atwood, B. 1987, ApJ, 319,709

Crane, P., Lambert, D. L., \& Sheffer, Y. 1995, ApJS, 99, 107

Crawford, I. A. 1989, MNRAS, 241, 575

Crawford, I. A. 1995, MNRAS, 277, 458

Diamond, P. J., Goss, W. M., Romney, J. D., et al. 1989, ApJ, 347, 302
Dieter, N. H., Welch, W. J., \& Romney, J. D. 1976, ApJ, 206, L113

Diplas, A., \& Savage, B. D. 1994, ApJS, 93, 211

Draine, B. T., \& Katz, N. 1986, ApJ, 392, 407

Faison, M. D., \& Goss, W. M. 2001, AJ, 121, 2706

Falgarone, E., \& Puget, J.-L. 1995, A\&A, 293, 840

Federman, S. R. 1982, ApJ, 257, 125

Federman, S. R., Strom, C. J., Lambert, D. L., et al. 1994, ApJ, 424, 772

Federman, S. R., Rawlings, J. M. C., Taylor, S. D., \& Williams, D. A. 1996, MNRAS, 279, L41

Frail, D. A., Weisberg, J. M., Cordes, J. M., \& Mathers, C. 1994, ApJ, 436, 144

Gredel, R. 1997, A\&A, 320, 929

Gredel, R., Pineau des Forêts, G., \& Federman, S. R. 2002, A\&A, 389, 993

Hennebelle, P., \& Pérault, M. 1999, A\&A, 351, 309

Heiles, C. 1997, ApJ, 481, 193

Herbig, G. H. 1999, PASP, 111, 809

Jenkins, E. B., \& Tripp, T. M. 2001, ApJS, 137, 297

Jenniskens, P., Ehrenfreund, P., \& Désert, F.-X. 1992, A\&A, 137, 297

Jenniskens, P., \& Désert, F.-X. 1994, A\&AS, 106, 39

Knauth, D. C., Federman, S. R., Pan, K., Yan, M., \& Lambert, D. L. 2001, ApJS, 135, 210

Krełowski, J., Ehrenfreund, P., Foing, B. H., et al. 1999, A\&A, 347, 235

Lauroesch, J. T., \& Meyer, D. M. 1999, ApJ, 519, L181

Le Petit, F., Boissé, P., Pineau des Forêts, G., et al. 2001, Proceedings of the XVII IAP Colloquium: Gaseous Matter in Galaxies and Intergalactic Space, 45

Lien, D. J. 1984, ApJ, 284, 578

Liszt, H., \& Lucas, R. 2000, A\&A, 333, 355

Marscher, A. P., Moore, E. M., \& Bania, T. M. 1993, ApJ, 419, L101

McCarthy, J. K., Sandiford, B. A., Boyd, D., \& Booth, J. 1993, PASP, 105,881

Meyer, D. M., Roth, K. C., \& Hawkins, I. 1989, ApJ, 343, L1

Meyer, D. M., \& Jura, M. 1985, ApJ, 297, 119

Miller, L. H. 1956, J. Amer. Stat. Ass., 47, 425

Moore, E. M., \& Marscher, A. P. 1995, ApJ, 452, 671

Pan, K., Federman, S. R., \& Welty, D. E. 2001, ApJ, 558, L105

Pineau des Forêts, G., Flower, D. R., Hartquist, T. W., \& Dalgarno, A. 1986, MNRAS, 220, 801

Roueff, E. 2001, Proceedings of the XVII IAP Colloquium: Gaseous Matter in Galaxies and Intergalactic Space, 37

Snow, T. P. 1993, ApJ, 402, L73

Spaans, M. 1995, Ph.D. Thesis, University of Leiden

Thoraval, S., Boissé, P., \& Stark, R. 1996, A\&A, 973, 312

Welty, D. E., \& Fitzpatrick, E. L. 2001, ApJ, 551, L175

White, R. E. 1984, ApJ, 284, 685 